\title{
Performance Evaluation of STHT based Phase Vocoder for Hearing Impaired
}

\author{
Vaishali R. Chopade, Mahesh T. Kolte and Anjali A. Shrivastav \\ Department of Electronics and Telecommunication Engineering, \\ Pimpri Chinchwad College of Engineering, Pune - 411044, Maharastra, India; \\ vaishalichopade1@gmail.com,mtkolte@yahoo.com, anjali.shrivastav@pccoepune.org
}

\begin{abstract}
Objectives: The performance of hearing aid largely depends on its ability to enhance the quality of speech. The objective of this paper is to find out the changes in time pattern of the speech signals which would help to resolve the issue of the temporal resolution. Methods/Statistical Analysis: Various Fourier transformation techniques are available for speech enhancement in hearing aid. This paper proposes Short Time Hartley transform (STHT) signal processing technique based phase vocoder for hearing impairment. Pitch shifting though time scaling is obtained by using phase vocoder. The proposed algorithm is implemented in MATLAB and verified with Vowel Consonant Vowel (VCV) data sets. Findings: In this paper, formants which are concentration of acoustic energy around a particular frequency are calculated for both STFT and STHT techniques and compared for performance evaluation using VCV data sets. The variation in pitch and power for different time scaling factors is observed which reflects the changes in time pattern of speech signals. The results obtained supports Short-Time Fourier transforms (STHT) signal processing technique to be effective for Phase vocoder performing time scaling and pitch shifting of audio as well as speech signals and can be a remedy for temporal resolution issue. Application/Improvements: As pitch shifting is observed towards both lower and higher frequency ranges, STHT can be a suitable processing technique, beneficial for hearing impaired people with sensorineural hearing loss.
\end{abstract}

Keywords: Hearing Impairment, and Sensorineural Hearing Loss, Phase Vocoder, Short Time Hartley Transform (STHT)

\section{Introduction}

Hearing impaired refers to individuals with hearing inability either partially or in total. As per World Health Organization (WHO), over $5 \%$ of the world's population which amounts to approximately 466 million people suffers from hearing loss and by 2050 over 900 million people are anticipated to fall prey to this disability 1 . Early detection and intervention are crucial to minimize the impact of hearing loss on the physical and mental state of Hearing Impaired.

Digital hearing aids come to the rescue of hearing impaired as it makes some sounds louder and audible so that a person with hearing loss can listen, communicate and participate more effectively in their daily chores. The digital hearing aids can be programmed with multiple programs that can be invoked by hearing impaired. These programs can reduce noise or amplify certain frequencies ${ }^{2}$. The hearing aid or hearing equipment is useful as facility to human being as per their needs. But this thing only is not the base of happiness of that human being. It will be supportive component to interact with family and society.

Industries (hearing aids) can be established on the base of needs. There will be an imbalance if it started on the basis of Profit frenzy. The needs, manufacturing, relations and balance should be compulsorily considerable points in this regards 3 . Hearing loss results in weakening of sensitivity to the sounds which otherwise

${ }^{*}$ Author for correspondence 
can be easily heard by a normal individual. The hard of hearing people are relatively insensitive to sound falling in the range of speech frequencies. For these hearing impaired people, the volume needs to be increased above the usual level for them to detect it. Vowels and consonants are integral part of speech. Various vowel sounds, which carry the emotions, are differentiated by unique sets of formant frequencies $\frac{4,5}{}$. The time varying Fourier transform [STFT] and Hartley transform [STHT], can be used for the processing of speech signals of hearing impaired which may overcome the drawbacks of the Fourier Transform $\frac{6,7}{}$. This paper introduces short time Hartley transform [STHT] for analysis of timevarying signals. Literature suggests Hartley transform to be better than Fourier transform since it involves only real operations. Also the transform kernel of Hartley in case of both forward and inverse transforms remains unchanged indicating that the same hardware built for forward transform computation can be used for inverse transform computing without amendments $\stackrel{8,9}{\text {. }}$.

\section{Short-Time Hartley Transform (STHT)}

Hartley transform is an integral transformation that uses only real values for conversion of data from time to frequency domain. The STHT removes imaginary numbers and only takes real numbers so it can be used in measurable quantity and it increases the computational complexity of the transformation ${ }^{10}$.

Hartley representation of time varying signal $f(t)$ in its one dimension is as shown in Equation 1.

$$
\operatorname{STHT}(t, \omega)=\int f(t) g(t) \operatorname{cas}(2 \Pi \omega t)
$$

Here ' $g$ ' and ' $\mathrm{l}$ ' represents window function and width of the window respectively.

Further, kernel in Equation 1 represents 45-degree phase shifted sine wave as shown in Equation 2.

$$
\operatorname{cas}(2 \Pi \omega t)=\cos (2 \Pi \omega t)+\sin (2 \Pi \omega t)
$$

\section{Phase Vocoder}

The phase vocoder modifies its short-time Fourier transforms for time scaling and pitches shifting of speech signals resulting in improved frequency estimates and thus leads to higher quality compared to time-domain time-scaling and pitch-shifting techniques. This digital signal processing algorithm, introduced by Flanagan and Golden in 1966, falls under category of analysissynthesis techniques and operates in three phases viz. Analysis, Transformation and Synthesis. It uses phase information for scaling frequency and time domains of speech signals. Based on the fundamental idea that pitch shifting and time stretching are reversible processes, phase vocoder stretches the original speech signal in time domain without making any changes in its original pitch. Short time Fourier transforms which is coded through the use of fast Fourier transform forms the crux of the phase vocoder. The phase vocoder is also used in computer music for its ability to expand and compress time and shift the pitch of audio ${ }^{9,10}$. This paper focuses on the implementation of phase vocoder algorithm using STHT technique.

\section{Implementation}

The proposed Phase Vocoder algorithm for time scale modification of speech signal is represented in the form of flowchart as shown in Figure 1 elaborates the flow of proposed algorithm where in for frequency transformation using STFT/STHT, first samples are broken into frames, then windowing function is applied to each of the frames and finally FFT is applied to windowed frame. In similar fashion, to acquire new framed signal at output using ISTFT/ISTHT, first each frame is collected from an STFT/STHT, N-point IFFT is applied to each windowed

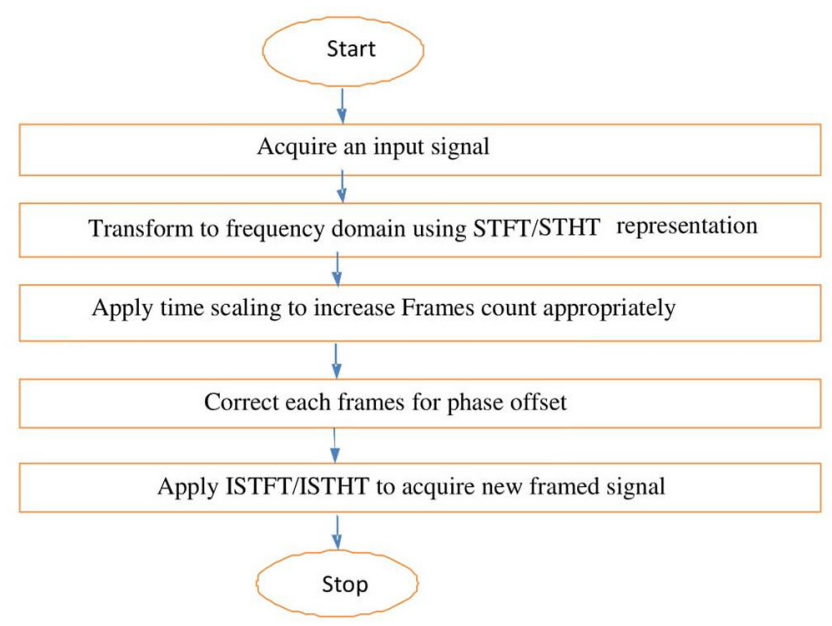

Figure 1. Flowchart of Proposed Phase Vocoder Algorithm. 


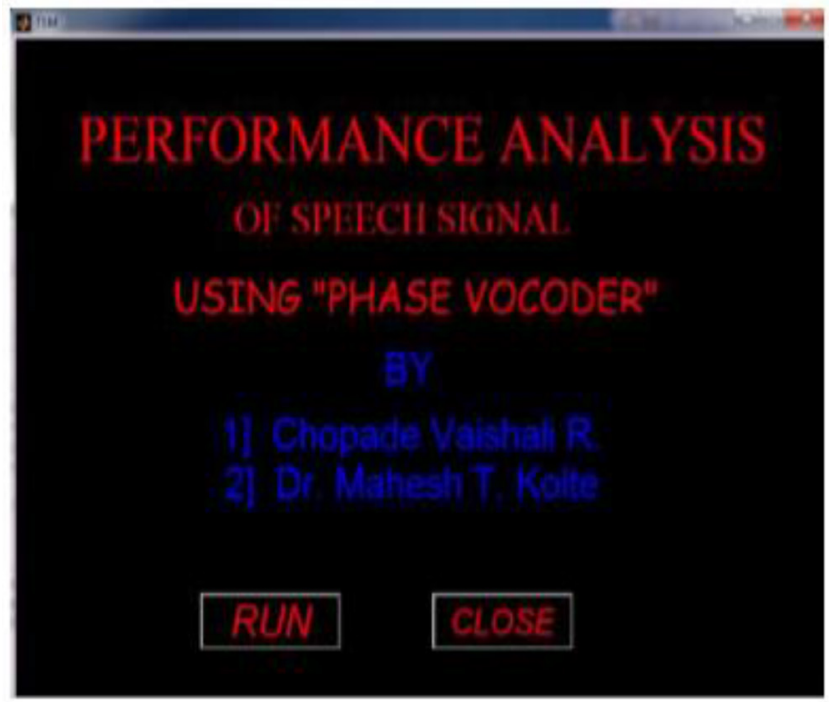

Figure 2. Welcome window of Designed Graphical User Interface.

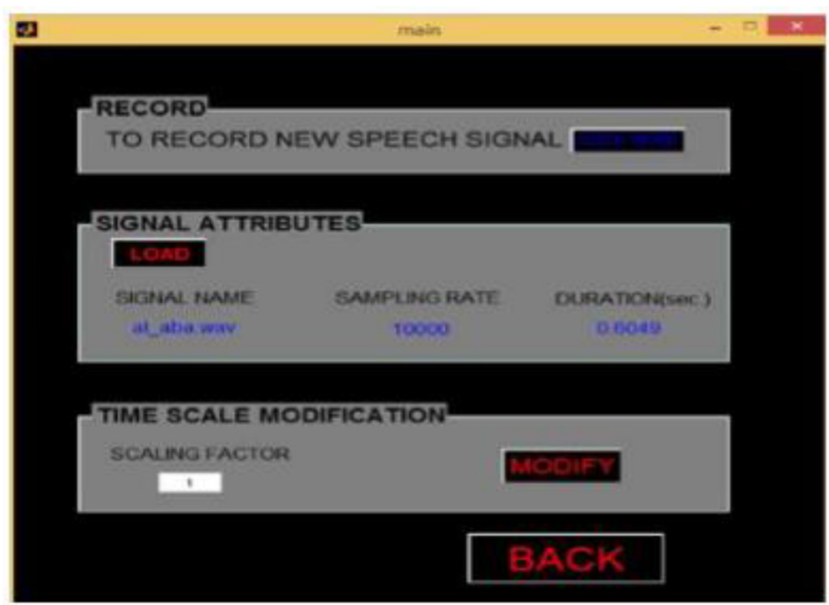

Figure 3. GUI providing options for signal recording and modification.

frame and finally new frames are staggered by hop and add overlap sections ${ }^{11}$. For execution of this proposed algorithm, Graphical User Interface is required to make necessary modifications in the original signal. MATLAB GUI development environment was used for creating the graphical interfaces. Figure 2 shows GUI which is used for execution of the algorithm. The GUI shown in Figure 3 provides LOAD and RECORD options to get a pre-recorded signal or record a new signal respectively. MODIFY option allows for modification in the original signal by varying the scaling factor $(\mathrm{SF})$ wherein $\mathrm{SF}=1$ indicates original signal whereas $\mathrm{SF}<1$ and $\mathrm{SF}>1$ leads to expansion and compression of original signal. Time

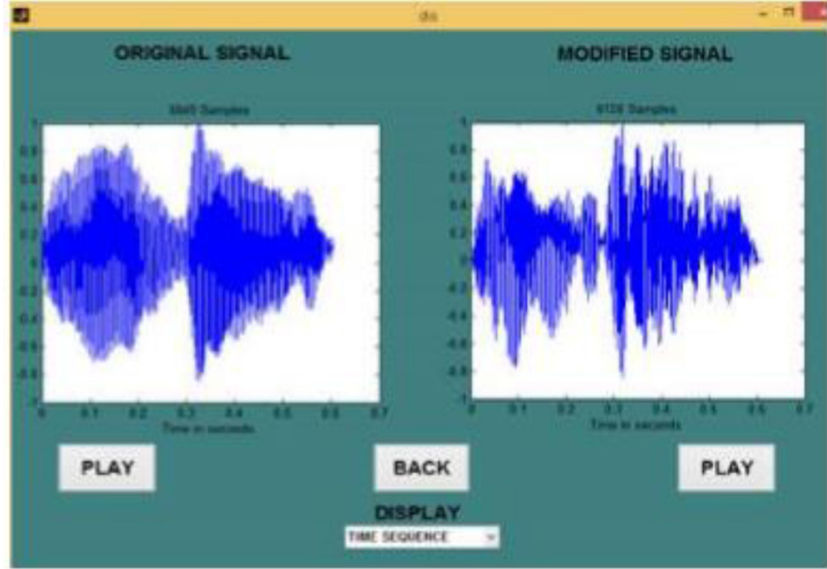

Figure 4. Time Sequence of original vs modified signal.

Sequence of original as well as modified signal is shown in two different panes of Figure 4. It can also display the signal using various other tools such as Spectrogram, Scalogram, Power Spectrum, etc.

\section{Results and Discussion}

The speech signal was given as input through the headphones. First the variations in the Pitch and Power of the original speech signal and signal modified using STFT and STHT signal processing technique were found as shown in Table 1. The pitch changes are noted for a data set of five VCV words using STFT and STHT techniques and observed pitch reduction was observed for ABA, AZA and ADA words using STFT and for ABA using STHT.

Thus hearing impaired people with sensor neural hearing loss who suffers high frequency loses can $b$ benefitted from this pitch shifting. Also reduction in power was observed for ABA and AGA words. Next the original signal and signal modified using STFT and STHT were observed based on their formant frequencies as shown in Table 2. The changes in time pattern of signal needed to measure temporal resolution has next been found by varying the scaling Factor $(\mathrm{K})$. Here the variations in the Pitch and Power of the original speech signal were detected by varying the scaling factor. Table 3 shows the variations in the Pitch and Power for various signals using different scaling factors wherein pitch and power variations are found for five different values of scaling factor (K) viz.0.89,0.9439,1,1.059 and 1.122. 
Table 1. Pitch and Power of VCV words modified using STFT and STHT

\begin{tabular}{|l|l|c|c|}
\hline Nature of Signal & Signals & $\begin{array}{l}\text { Pitch Contour } \\
\text { (in Hz) }\end{array}$ & $\begin{array}{l}\text { Power (in } \\
\text { dB) }\end{array}$ \\
\hline Original & al_aba & 200 & 62.97 \\
\hline Modified STFT & aba_s & 195 & 59.67 \\
\hline Modified STHT & aba_h & 191 & 57.31 \\
\hline Original & al_ada & 193 & 62.57 \\
\hline Modified STFT & ada_s & 187 & 56.51 \\
\hline Modified STHT & ada_h & 195 & 60.92 \\
\hline Original & arp_aga & 201 & 51.00 \\
\hline Modified STFT & aga_s & 203 & 49.87 \\
\hline Modified STHT & aga_h & 200 & 48.90 \\
\hline Original & al_afa & 196 & 55.58 \\
\hline Modified STFT & afa_s & 197 & 49.08 \\
\hline Modified STHT & afa_h & 199 & 55.74 \\
\hline Original & al_aza & 183 & 52.93 \\
\hline Modified STFT & aza_s & 163 & 46.77 \\
\hline Modified STHT & aza_h & 213 & 48.48 \\
\hline
\end{tabular}

Table 2. Formant Frequencies for VCV words modified using STFT and STHT

\begin{tabular}{|l|l|l|}
\hline Name of signals & Signals & Formant Frequencies \\
\hline Original & al_aba & 219.23993 .142638 .533577 .69 \\
\hline Modified STFT & aba_s & 201.09988 .862677 .373723 .87 \\
\hline Modified STHT & aba_h & 322.591028 .372657 .203951 .83 \\
\hline Original & al_ada & 227.231938 .682970 .853967 .15 \\
\hline Modified STFT & ada_s & 197.241918 .623654 .314599 .00 \\
\hline Modified STHT & ada_h & 228.691817 .482101 .603599 .15 \\
\hline Original & arp_aga & 776.341473 .162400 .344444 .65 \\
\hline Modified STFT & aga_s & 593.751471 .972558 .534500 .81 \\
\hline Modified STHT & aga_h & 563.751436 .112466 .064453 .81 \\
\hline Original & al_afa & 202.57945 .972782 .513978 .53 \\
\hline Modified STFT & afa_s & 216.81971 .182830 .893865 .86 \\
\hline Modified STHT & afa_h & 195.191017 .782695 .153911 .81 \\
\hline Original & al_aza & 202.671933 .553186 .034186 .07 \\
\hline Modified STFT & aza_s & 298.541702 .653495 .264244 .74 \\
\hline Modified STHT & aza_h & 254.331649 .873332 .224202 .98 \\
\hline
\end{tabular}

The variation in original and modified signals has been observed for a set of 7 different signals using STFT and STHT technique.
Table 3 and 4 indicates that ABA and AZA data sets show increase in frequency using STHT while ADA, AGA and AFA shows lowering in frequency for few scaling factor values. Increase in power is observed for $\mathrm{ABA}, \mathrm{ADA}$ and AZA data sets in signals modified using STHT technique.

From the results, it is evident that the ABA signal shows increase in frequency ranging between 3700 $\mathrm{Hz}$ to $4500 \mathrm{~Hz}$ and AZA shows lowering in frequency ranging between 250 to $300 \mathrm{~Hz}$. The original signals ranges between $800 \mathrm{~Hz}$ to $2200 \mathrm{~Hz}$ frequency while the modified signals ranges between $2700 \mathrm{~Hz}$ to $4700 \mathrm{~Hz}$ frequency for ABA and AZA data sets indicating STHT to be a suitable candidate for Phase vocoder for digital hearing aids.

Table 3. Variation in Pitch for varying scaling factors

\begin{tabular}{|c|c|c|c|c|c|c|}
\hline \multirow{2}{*}{$\begin{array}{l}\text { Sr. } \\
\text { No. }\end{array}$} & \multirow[t]{2}{*}{ Signals } & \multicolumn{5}{|c|}{$\operatorname{Pitch}($ in $\mathrm{Hz})$} \\
\hline & & $\mathrm{k}-0.89$ & $\mathrm{k}=0.9439$ & $\mathrm{k}=1$ & $\mathrm{k}=1.059$ & $\mathrm{k}=1.122$ \\
\hline \multirow[t]{3}{*}{1} & al_aba & 198 & 201 & 200 & 195 & 191 \\
\hline & aba_s & 198 & 201 & 195 & 191 & 191 \\
\hline & aba_h & 200 & 201 & 191 & 200 & 200 \\
\hline \multirow[t]{3}{*}{2} & al_ada & 199 & 197 & 193 & 221 & 216 \\
\hline & ada_s & 199 & 197 & 187 & 221 & 216 \\
\hline & ada_h & 198 & 195 & 195 & 214 & 216 \\
\hline \multirow[t]{3}{*}{3} & arp_aga & 203 & 205 & 201 & 203 & 200 \\
\hline & aga_s & 203 & 205 & 203 & 203 & 200 \\
\hline & aga_h & 202 & 205 & 200 & 200 & 191 \\
\hline \multirow[t]{3}{*}{4} & al_afa & 199 & 191 & 196 & 197 & 203 \\
\hline & afa_s & 199 & 191 & 197 & 197 & 203 \\
\hline & afa_h & 198 & 201 & 199 & 199 & 209 \\
\hline \multirow[t]{3}{*}{5} & al_aza & 191 & 187 & 183 & 176 & 130 \\
\hline & aza_s & 191 & 187 & 163 & 176 & 130 \\
\hline & aza_h & 201 & 183 & 213 & 212 & 130 \\
\hline \multirow[t]{3}{*}{6} & $1 \mathrm{khz}$ & 100 & 100 & 100 & 220 & 220 \\
\hline & 1khz_s & 100 & 100 & 100 & 220 & 220 \\
\hline & 1khz_h & 100 & 100 & 100 & 147 & 220 \\
\hline \multirow[t]{3}{*}{7} & $440 \mathrm{hz}$ & 227 & 227 & 227 & 333 & 333 \\
\hline & 440hz_s & 227 & 227 & 227 & 333 & 333 \\
\hline & 440hz_h & 232 & 232 & 234 & 254 & 338 \\
\hline
\end{tabular}


Table 4. Variation in Power for varying scaling factors

\begin{tabular}{|c|c|c|c|c|c|c|}
\hline \multirow{2}{*}{$\begin{array}{l}\text { Sr. } \\
\text { No. }\end{array}$} & \multirow[t]{2}{*}{ Signals } & \multicolumn{5}{|c|}{ Power(in dB) } \\
\hline & & $\mathrm{k}-0.89$ & $\mathrm{k}=0.9439$ & $\mathrm{k}=1$ & $\mathrm{k}=1.059$ & $\mathrm{k}=1.122$ \\
\hline \multirow[t]{3}{*}{1} & al_aba & 67.84 & 66.56 & 62.97 & 62.61 & 58.42 \\
\hline & aba_s & 67.84 & 66.56 & 59.67 & 62.61 & 58.42 \\
\hline & aba_h & 67.69 & 66.56 & 57.31 & 62.67 & 53.27 \\
\hline \multirow[t]{3}{*}{2} & al_ada & 69.57 & 64.84 & 62.57 & 49.87 & 59.81 \\
\hline & ada_s & 69.57 & 64.84 & 56.51 & 49.87 & 59.81 \\
\hline & ada_h & 63.37 & 62.42 & 60.92 & 48.9 & 59.81 \\
\hline \multirow[t]{3}{*}{3} & arp_aga & 54.94 & 51.66 & 51 & 49.08 & 49.61 \\
\hline & aga_s & 54.94 & 51.66 & 49.87 & 49.08 & 49.61 \\
\hline & aga_h & 52.31 & 51.66 & 48.9 & 55.74 & 45.02 \\
\hline \multirow[t]{3}{*}{4} & al_afa & 65.53 & 56.66 & 55.58 & 46.87 & 45.71 \\
\hline & afa_s & 65.53 & 56.66 & 49.08 & 46.87 & 45.71 \\
\hline & afa_h & 62.63 & 61.38 & 55.74 & 48.55 & 47.26 \\
\hline \multirow[t]{3}{*}{5} & al_aza & 56.87 & 53.41 & 52.93 & 59.67 & 45.25 \\
\hline & aza_s & 56.87 & 53.41 & 46.77 & 57.31 & 45.25 \\
\hline & aza_h & 56.67 & 53.38 & 48.48 & 57.31 & 45.25 \\
\hline \multirow[t]{3}{*}{6} & $1 \mathrm{khz}$ & 63.26 & 56.86 & 56.86 & 56.84 & 56.84 \\
\hline & 1khz_s & 59.31 & 56.86 & 56.86 & 56.84 & 56.84 \\
\hline & 1khz_h & 63.26 & 56.92 & 56.8 & 55.04 & 54.92 \\
\hline \multirow[t]{3}{*}{7} & $440 \mathrm{hz}$ & 63.26 & 63.26 & 63.26 & 63.24 & 63.24 \\
\hline & 440hz_s & 63.26 & 63.26 & 63.26 & 63.24 & 63.24 \\
\hline & 440hz_h & 59.31 & 60.19 & 62.83 & 57.92 & 54.63 \\
\hline
\end{tabular}

\section{Conclusion}

This paper highlights the use of Short-Time Fourier transforms (STHT) signal processing technique which is found effective for Phase vocoder performing time scaling and pitch shifting of audio as well as speech signals. The performance has been evaluated taking into consideration various parameters like pitch contour, power and formant frequencies for original signals and signals modified using STFT and STHT techniques. Also the variation in pitch and power for varying scaling factors has been observed which reflects the changes in time pattern of signal and thus helps to remedy temporal resolution. As pitch shifting is observed towards both lower and higher frequency ranges, STHT can be a suitable processing technique, beneficial for hearing impaired people with sensorineural hearing loss.

\section{References}

1. Deafness and hearing loss. Available from: http://www.who. int/newsroom/factsheets/detail/deafness-and-hearingloss. Date accessed: 15/03/2018.

2. Neelam Joshi, Kolte MT. Digital Hearing Aid - A Review. International Journal of Innovative Research in Electrical, Electronics, Instrumentation and Control Engineering. 2013; 1(8):369-72.

3. Nagraj A. Samadhanatmak Bhotikvad, 3rd edn. Jeevan Vidta Prakashan, Amarkantak, Madhya Pradesh, India, 2009.

4. Kolte MT, Chaudhari DS. Evaluation of Speech Processing Schemes for Improving Perception of Sensorineural Hearing Impaired. Current Science. 2010; 98(5):613-15.

5. Kolte MT. Experimental Evaluation Using Signal Processing Techniques for Aids Used by Hearing Impaired. Doctoral dissertation, Sant Gadge baba Amravati University, India. 2008.

6. Bousselmi S, Aloui N, Cherif A. DSP Real-Time implementation of an Audio compression algorithm by using the Fast Hartley transforms. International Journal of Advanced Computer Science and Applications. 2017; 8(4):472-7.

7. Liu JC, Lin TP. Short-time Hartley transform. Proceedings of IEE Radar and Signal Processing. 1993; p. 171-4. https:// doi.org/10.1049/ip-f-2.1993.0024

8. Sundararajan N, Ebrahimi A, Va-sudha N. Two Dimensional Short Time Hartley Transform. SQU Journal for Science. 2016; 21(1):41-7. https://doi.org/10.24200/squjs.vol21iss1 pp41-47

9. Laroche J, Dolson M. Improved phase vocoder time-scale modification of audio. IEEE Transaction Speech and Audio Processing. 1999; 3:323-32. https://doi.org/10.1109/89.759041

10. Dolson M. The phase vocoder: A tutorial. Computer Music Journal. 1986; 10(4):14-27. https://doi.org/10.2307/3680093

11. Vaishali R. Chopade, Dr. Mahesh T. Kolte. STHT based Speech Signal Processing using phase vocoder, 2018, August, ICCUBEA 18. 